\title{
Probing Spatial Locality in Ionic Liquids with the Grand Canonical Adaptive Resolution Molecular Dynamics Technique
}

B. Shadrack Jabes, ${ }^{1}$ C. Krekeler, ${ }^{1}$ R. Klein, ${ }^{1}$ and L. Delle Site ${ }^{1,}$ a)

Institute for Mathematics, Freie Universitat Berlin, D-14195 Berlin, Germany

(Dated: 21 June 2021)

We employ the Grand Canonical Adaptive Resolution Molecular Dynamics Technique (GC-AdResS) to test the spatial locality of the 1-ethyl 3-methyl imidazolium chloride liquid. In GC-AdResS atomistic details are kept only in an open sub-region of the system while the environment is treated at coarse-grained level, thus if spatial quantities calculated in such a sub-region agree with the equivalent quantities calculated in a full atomistic simulation then the atomistic degrees of freedom outside the sub-region play a negligible role. The size of the sub-region fixes the degree of spatial locality of a certain quantity. We show that even for sub-regions whose radius corresponds to the size of a few molecules, spatial properties are reasonably reproduced thus suggesting a higher degree of spatial locality, a hypothesis put forward also by other researchers and that seems to play an important role for the characterization of fundamental properties of a large class of ionic liquids.

a) Author for correspondence:luigi.dellesite@fu-berlin-de (L.D.S) 


\section{INTRODUCTION}

Ionic liquids (ILs) are a new class of air- and water-stable organic salts whose melting point is around room temperature ${ }^{1}$. They have the ionic nature of inorganic solvents and organic nature of organic solvents. Because of this dual or amphiphilic nature of ions, they inherit both the solvent characteristics and possess even more excellent properties, such as high electrical conductivity, improved electrical and thermal stability, good solvation ability, negligible vapor pressure, non-flammability, to mention a few $\underline{2}^{2} \underline{3}$. When the balance between the dual nature of ILs is altered by chemical modifications of the polar or apolar components or charge of the ions, an exponentially large number of ILs with a wide range of physicochemical properties such as viscosity, catalytic activity, or solvation can be generated. Ionic liquids engineered at the molecular level to have specific physical and chemical properties, can be employed for a range of designated applications $\underline{4}, \underline{5}$ : ionic liquids as drugs $\underline{6}, \underline{7}$, as batteries and super-capacitors for solving energy demands of the environment $\stackrel{-11}{-11}$, to capture greenhouse gases, to dissolve proteins $\frac{12,13}{13}$ and cellulose $\underline{14}, 15$.

The design and testing of new liquids, however, given the innumerable chemical combinations of anions and cations, rises the problem of their optimal design since using experiments is a rather time consuming and unfeasible strategy ${ }^{16}$. Therefore, many studies involve the use of computer simulations so as to understand and predict such properties in silico and then drive experiments, and the present paper contributes to the development of suitable simulation technology.

The key question that chemical engineers must face for ILs concerns the possibility of linking, in a rational way, local structural organization or microscopic interactions and the large scale physico-chemical behavior. For example: the separation of fission products such as $\operatorname{Cs}(+)$ and $\operatorname{Sr}(2+)$ from aqueous solutions using chemically modified ionic liquids are influenced by the structures of imidazolium cations attached to each other by covalent bonding 17 . The hydroxyl- and carboxyl-functionalised ILs exhibit molecular recognition characteristics that can be used to separate even homologue zwitterionic phospholipid species out of homogeneous mixtures. These selectivity characteristics can be augmented by the tendency of ILs to form meso-phases, polar-apolar alternations at the nanoscale $\underline{\underline{18}} \underline{22}$. Thus a profound understanding of local structures of ILs in the bulk or at interfaces and the relationship between local structures and thermodynamic properties is mandatory for choosing an optimal 
combination of ILs to obtain the desired properties on demand.

Several computational studies have been carried out to characterize the locality in ILs, that is, how a molecule and its immediate surroundings respond to changes occurring on the global mesoscopic scale, e.g., the hydrogen bonding network of the bulk or other properties which typically occur on a larger scale $\underline{19,21,23}-\underline{25}$. In particular, the structural locality and its relation to the governing interactions was actually either studied at a quantum level or at a coarse grained level. For example: Ab initio simulation studies suggest that the electric dipole moment of the ions is highly local, meaning that it is sufficient to know only the details of the very immediate environment ${ }^{25}$. At the same time, coarse-grained simulations have been used to study the heterogeneous structural ordering and its dynamics providing a very simplified model of the bulk 19,21 .

For the studies reported above, however, one must consider that ab initio techniques are limited to small gas phase systems of relatively small samples of liquids, and are thus not yet appropriate for the exhaustive sampling of large scale liquid properties. Coarse-grained models, in turn, use a simplified approach that ignores the role of atomistic degrees of freedom and thus allows the chemical nature of the anions and cations to enter only marginally. A reasonable compromise for linking the atomistic and mesoscopic scale is the use of classical Molecular Dynamics (MD) simulations in which the only limiting requirement is the necessity of long simulations times for a proper statistical sampling. In present work, in particular, we employ classical molecular dynamics within the context of the Adaptive Resolution Simulation approach (AdResS) ${ }^{26,27}$ in its Grand Canonical version (GC-AdResS) ${ }^{28-30}$.

GC-AdResS is a multi-scale method that allows the molecules to change their resolution on the fly from high-resolution to lower resolution region and vice versa. The higher resolution region corresponds to a local region in space that is defined by an atomistically resolved model while the surrounding bulk requires a less accurate model (coarse-grained), sufficient for reproducing only the large scale thermodynamic properties. In essence, the region of interest inside of which atomistic details are relevant is embedded in a thermodynamic bath with which it exchanges energy and particles in a Grand Canonical fashion. Relevant technical details of GC-AdResS are reported in the technical section; for a general overview of this method and related ones see Ref. 31 .

The key aspect of GC-AdResS that is important in the context of the present work is its capability to be used as an analysis tool of MD simulations to identify, for a given property, 
the relevant atomistic degrees of freedom of the system for a certain quantity or property of interest. In fact, a systematic variation of the size of the atomistically resolved region implies a systematic variation of the number of atomistic degrees of freedom. Next, suppose we compare a given property calculated in the atomistic region of GC-AdResS with the same property calculated in an equivalent sub-region of a full atomistic simulation. By such a comparison it is possible to draw unambiguous conclusions regarding the influence of the explicit atomistic degrees of freedom residing outside of the sub-region on our property of interest. In fact if the property calculated shows the same behavior in both cases, it implies that the role of the explicit atomistic degrees of freedom outside the considered sub-region is negligible. This conclusion in turn implies that the minimum size of a sub-region where GC-AdResS and a full atomistic simulation agree (for a certain quantity) defines the degree of spatial locality of such quantity w.r.t. the atomistic degrees of freedom.

AdResS as a tool to identify spatial locality in complex molecular systems has been already successfully applied in past and recent work (see Refs. $\underline{32}, 33$ ). In the context of ILs, coming back to the earlier discussion about the necessity of linking specific chemical structures to large scale properties for an optimal design and combination of anions and cations, the GC-AdResS analysis can play a relevant role. In fact, its aim is exactly the identification of the minimal high resolution islands of anions and cations which, without loss of accuracy, can be embedded in the larger bulk with its mesoscopic properties obtained at lower resolution. In this work we provide a practical example for a relevant IL, [Emim] [Cl]. Of particular interest is the result that the degree of locality can be much higher than one may expect at an intuitive level. The satisfactory technical applicability of GC-AdResS to ILs, employing charged or uncharged spherical particles as coarse-grained models, has already been proven, in a rather detailed manner, in preceding work ${ }^{34}$. Therefore, this paper represents an extension of the technical implementation to the use as a multi-scale analysis tool. A discussion regarding the utility of information on locality for chemical design and molecular modeling in ILs concludes the paper. 


\section{COMPUTATIONAL DETAILS}

\section{A. Method}

We employ the Adaptive Resolution Simulation Scheme (AdResS) 26.27 for molecular dynamics in its more advanced version in the Grand Canonical interpretation (GCAdResS) ${ }^{28,29}$. AdResS is a multi-resolution simulation method that links two regions of a simulation box having different resolution, regions:1. atomistic (AT) and 2. a coarsegrained ( $\mathrm{CG}$ ) system, and it allows the free exchange of particles between the two regions through a coupling transition region (HY) where molecules have space-dependent hybrid atomistic/coarse-grained resolution. In the hybrid region, the force between two molecules $\alpha$ and $\beta$ is computed via a space-dependent interpolation formula and is written as

$$
F_{\alpha \beta}=w\left(X_{\alpha}\right) w\left(X_{\beta}\right) F_{\alpha \beta}^{A T}+\left[1-w\left(X_{\alpha}\right) w\left(X_{\beta}\right)\right] F_{\alpha \beta}^{C G}
$$

here, $F_{\alpha \beta}^{A T}$ is the force between the particles derived using the atomistic potential and $F_{\alpha \beta}^{C G}$ is the force derived from the $\mathrm{CG}$ potential. $w(x)$ is the interpolating switch and is defined as:

$$
\left\{\begin{array}{ll}
1 & x<d_{A T} \\
\cos ^{2}\left[\pi 2\left(d_{\Delta}\right)\left(x-d_{A T}\right)\right] & d_{A T}<x<d_{A T}+d_{\Delta} \\
0 & d_{A T}+d_{\Delta}<x
\end{array}\right\}
$$

where, $d_{A T}$ and $d_{\Delta}$ are the spatial extent of the atomistic region and hybrid regions respectively (see Fig (1). The weighting function (shown in yellow line) smoothly goes from 0 to 1 in the hybrid region and allows the coarse grained particles to change their resolution into an atomistic molecule and vice versa. This minimal set up has been shown already sufficient for performing accurate simulations in both the atomistic and coarse-grained region (see e.g. $\left.\frac{35}{3}\right)$.

However, further technical and conceptual developments led to the definition of a thermodynamic force in the transition region which assures a priori proper thermodynamic

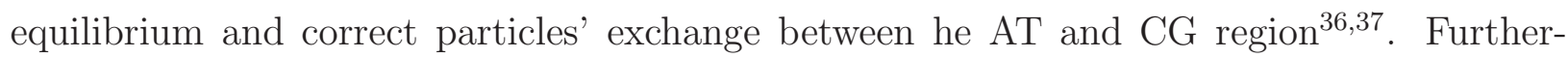
more, it has been shown that for the coarse-grained model it is sufficient to develop models that reproduce only density and temperature, that is macroscopic thermodynamics, so that AdResS is re-framed within the Grand Canonical approach $\frac{28,29}{2}$, or even further, within the 
more general Grand Ensemble idea of open boundary systems ${ }^{30,31,38}$. For the specific study in this work, the atomistic region is modelled as a sphere of radius $d_{a t}$ and is surrounded by a spherical hybrid region of width $d_{h y}$ and the remainder of the box is the CG region that serves as a particle reservoir for the AT region; see the pictorial representation in Fig.1. The force field parameters of the atomistic model and of the coarse-grained model are reported in sections $\llbracket \mathrm{B}$ and $\coprod \mathrm{C}$.

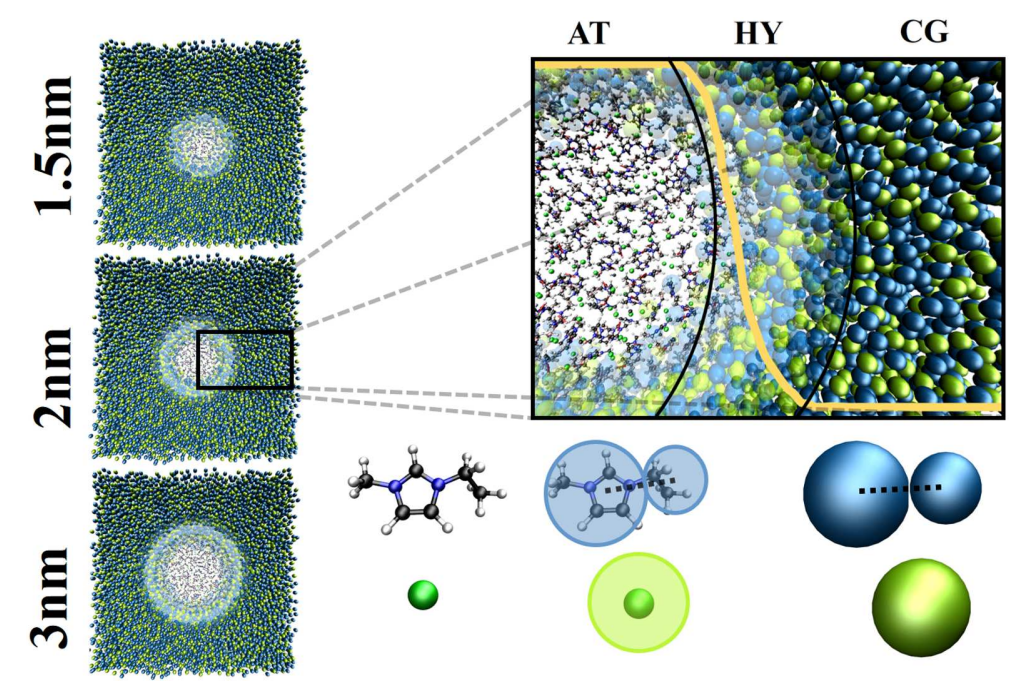

FIG. 1. (Right column)Schematic representation of the GC-AdResS scheme; CG, HY and AT represents the coarse-grained region, hybrid region and the atomistic region respectively. The CG model consists of one sphere for the imidazolium ring and one sphere for the alkyl side chain (cation) and a sphere for the anion which has an effective size different from the atomistic representation. Within the AT and CG regions the force acting between two molecules is derived from the respective potentials. However, in the HY region, the forces are obtained by a space dependent interpolation scheme in which a weighting function $\mathrm{w}(\mathrm{x})$ (shown in yellow) allows a smooth transition from one resolution to another. In the left column we show three AdResS simulations used in the study with three atomistic resolutions spanning from $1.5 \mathrm{~nm}$ to $3 \mathrm{~nm}$ for the radius of the atomistic region

\section{B. Models}

The full atomistic force field parameters for modeling 1-ethyl 3-methyl imidazolium chloride were taken from a study by Dommert et al ${ }^{39}$, in particular such model considers reduced/scaled charges of $+0.8 \mathrm{e}$ and $-0.8 \mathrm{e}$ instead of the full formal charges +1.0 e and $-1.0 \mathrm{e}$. In the coarse-grained region, ILs are modelled as charged/neutral spherical beads, i.e. one sphere for the imidazolium ring, bonded to one sphere for the alkyl side chain, for the cation and a sphere with an effective excluded volume, consistent with the CG model of the cation, 
for the anion (see Fig (1). The interaction parameters are developed using a straightforward Inverse Boltzmann Iterative procedure (IBI). Otherwise care must be taken in deriving the generic CG model because a charge neutral representation of coarse-grained bead for ILs would result in gaining artificial electrostatic interactions instantaneously in the atomistic and the reservoir regions. The IBI procedure employed in this study takes into account the corresponding charge in the coarse-grained model during the iterative process 34 .

\section{Technical details}

All the simulations were performed using GROMACS package ${ }^{40}$. We set up two systems, the first one (400 ion pairs) was used to derive two coarse grain potentials, which we then transferred to the larger system (19968 ion pairs). We optimized both systems using full atomistic NPT calculations. The simulation temperature was set to 400K, the time step was $2 \mathrm{fs}$ and the electrostatic interactions were calculated through the particle mesh Ewald (PME) technique. For the first 5 ns, we used Berendsen barostat, $\stackrel{41}{a}$ after that we switched to the Parrinello-Rahman barostat 2 . for the following 5ns. We monitored the box size and considered that we have reached convergence, when the changes in the box length were of the order of $0.0001 \mathrm{~nm}$. For 400 and 19968 ion pairs we obtained a cubic box with $4.31080 \mathrm{~nm}$ and $15.81094 \mathrm{~nm}$ respectively. Furthermore, the radial distribution function were obtained after $20 \mathrm{~ns}$ full atomistic NVT simulations at $380 \mathrm{~K}$ and with $2 \mathrm{fs}$ timesteps. The 400 ion pairs configuration was used to derive the two coarse-grained models. We used an inverse Boltzmann iteration, IBI, procedure $\frac{43}{3}$ to reproduce the radial distribution functions of the full atomistic target systems (for charged model see Fig. 3,5 and for the neutral model see Fig. 668). After we derive these tabulated potentials, we used the configuration from the 19968 ion pairs to set up the GC-AdResS system. We vary the resolution of the atomistic region in the GC-AdResS simulation from $1.3 \mathrm{~nm}$ - 3nm in order to look at the essential degrees of freedom that are responsible to capture the structural properties of the system similar to the full atomistic simulations. This atomistic region is bordered by hybrid regions of length $2 \mathrm{~nm}$, while the remaining length corresponds to the coarse-graining region. As in standard AdResS simulations, a Langevin thermostat is used with $\Gamma=5 p s^{-1}$. The electrostatic interactions, as usually done in AdResS, were treated by the generalized reaction field method with a self-consistent dielectric constant as calculated by GROMACS $\stackrel{44}{ }$. The 
use of the charged coarse-grained model implied that the reaction field method is also used in the coarse-graining region where it applies in the same way as in the atomistic region. The thermodynamic force through the iterative procedure was considered converged after 100 iterations, sufficient to reach an accuracy of $<5 \%$ particle density and for the ion-ion radial distribution functions ( compared to the full atomistic of the reference).

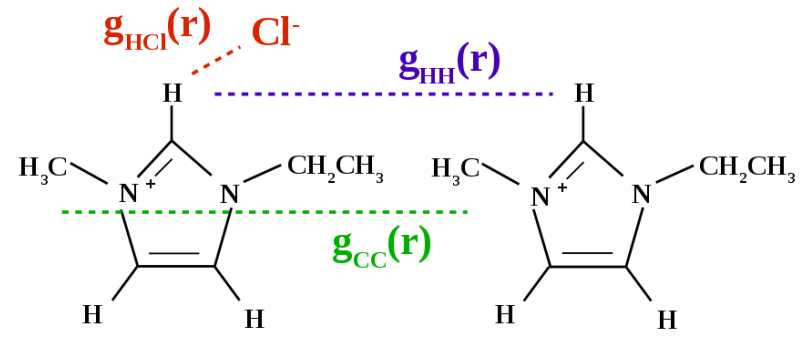

FIG. 2. Pictorial representation of the atom types corresponding to the radial distribution profile for the emim chloride system. The center of mass of the cations and anions are represented as CAT, ANN or Cl respectively. The carbon (C), of the methyl group is also represented as CAA.

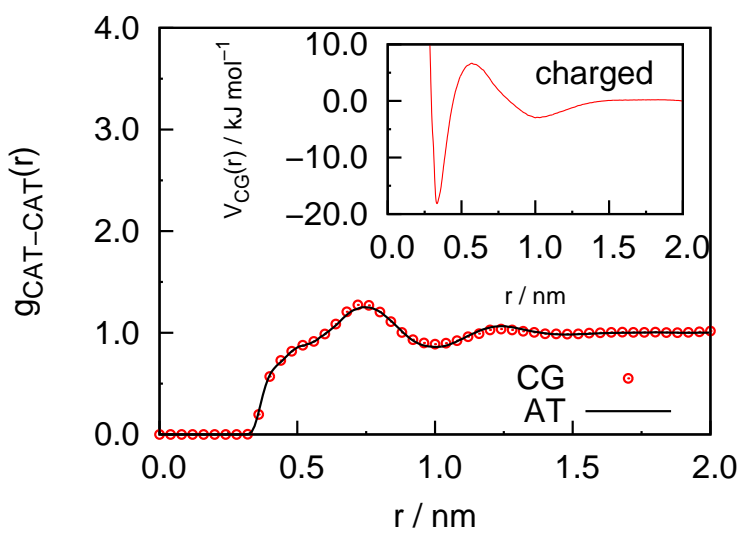

FIG. 3. The cation-cation radial distribution profile obtained from atomistic simulation (AT) and coarse-grained (CG) simulation as a function of distance $r$. The $\mathrm{CG}$ simulation employs the IBI potential for the charged CG model (inset).

\section{RESULTS AND DISCUSSION}

The GC-AdResS analysis involves two stages, the first concerns a careful check that necessary conditions of physical consistency are fulfilled, the second concerns the analysis of spatial locality done through quantities which capture the essence of the statistical properties of a system. In the first stage we check that the particle number density of the AdResS system 


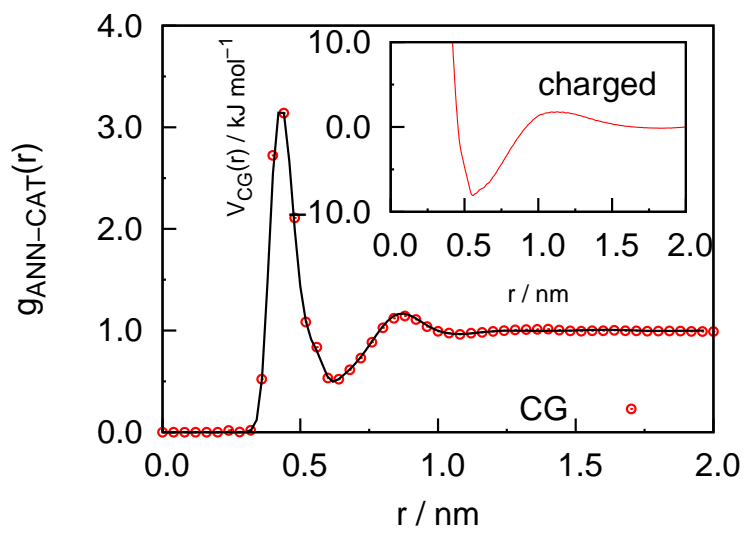

FIG. 4. The anion-cation radial distribution profile obtained from atomistic simulation (AT) and coarse-grained (CG) simulation as a function of distance $r$. The CG simulation employs the IBI potential for the charged CG model (inset).

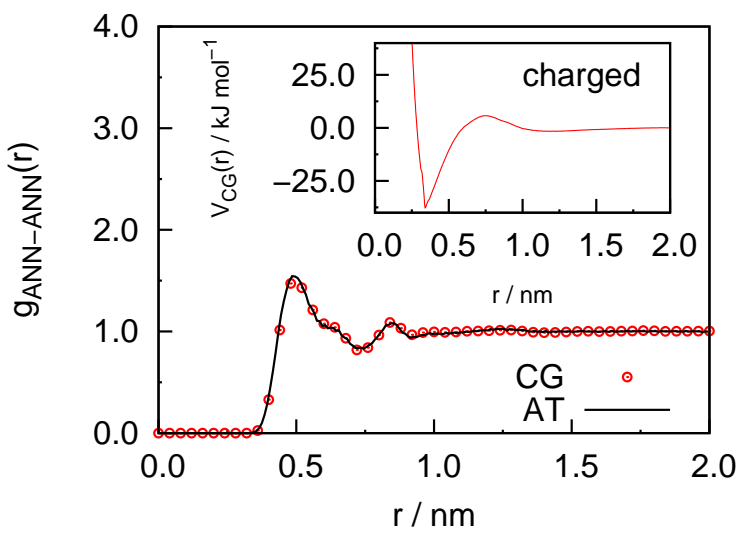

FIG. 5. The anion-anion radial distribution profile obtained from atomistic simulation (AT) and coarse-grained (CG) simulation as a function of distance $r$. The $\mathrm{CG}$ simulation employs the IBI potential for the charged CG model (inset).

agrees well (within a chosen accuracy, usually with $3-5 \%$, which has been already shown in previous work to be satisfactory); if this condition would not hold than we can obviously not have the same thermodynamic condition of the reference full atomistic system. Next we must check that the particle number probability distribution function of the atomistic region agrees well (once again within the chosen accuracy) with the same quantity calculated in the sub-region of a full atomistic simulation with the same size of the atomistic region considered in GC-AdResS. Such a quantity expresses the behavior of the particle number fluctuation and thus assures that the exchange of matter with the large reservoir occurs in the correct way. Finally, in order to assure that artificial structural effects due to the hybrid 


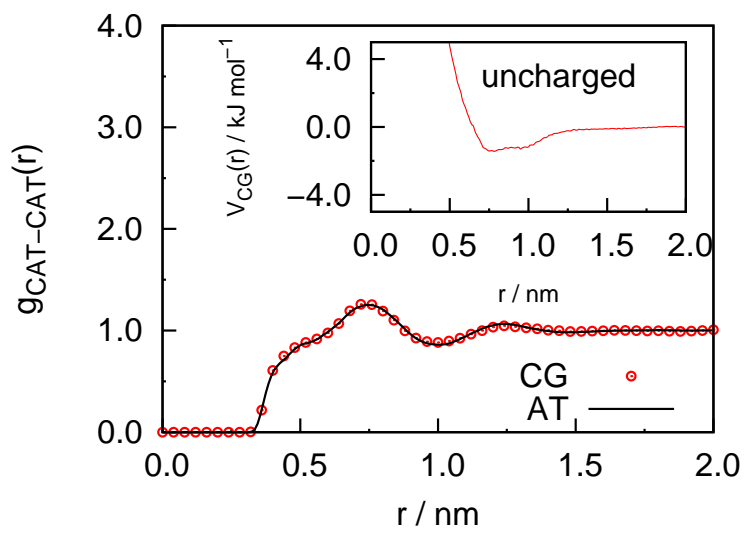

FIG. 6. The cation-cation radial distribution profile obtained from atomistic simulation (AT) and coarse-grained (CG) simulation as a function of distance $r$. The CG simulation employs the IBI potential for the uncharged CG model (inset).

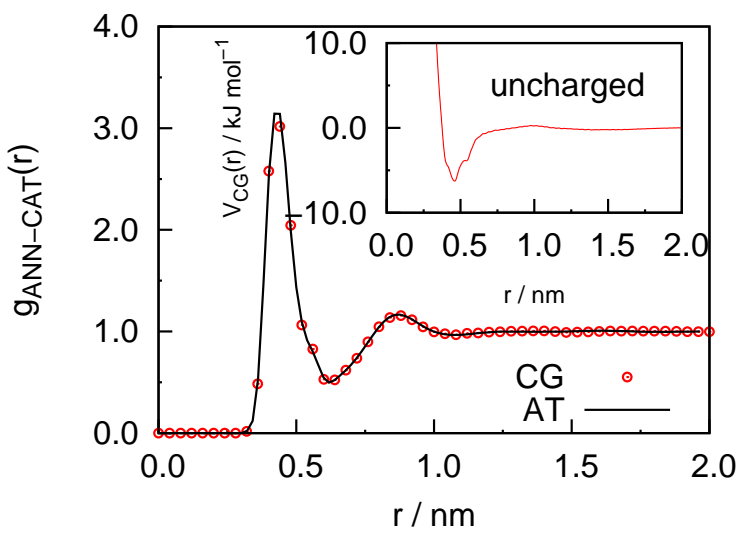

FIG. 7. The anion-cation radial distribution profile obtained from atomistic simulation (AT) and coarse-grained (CG) simulation as a function of distance $r$. The CG simulation employs the IBI potential for the uncharged CG model (inset).

region are negligible in the atomistic region, we calculate the probability distribution of an order parameter and compare with the equivalent calculated in the sub-region of the full atomistic region. The order parameter is system specific, while the other two quantities are universal; together they significantly characterize the system under investigation.

Once we are assured about the proper thermodynamic and statistical behavior of the atomistic region, we proceed with the analysis of the locality. For such an analysis we consider several radial distribution functions calculated in the atomistic region and compare them with the equivalent functions in the corresponding sub-region of the full atomistic system. In fact radial distribution functions represent the $3 N$-probability distribution func- 


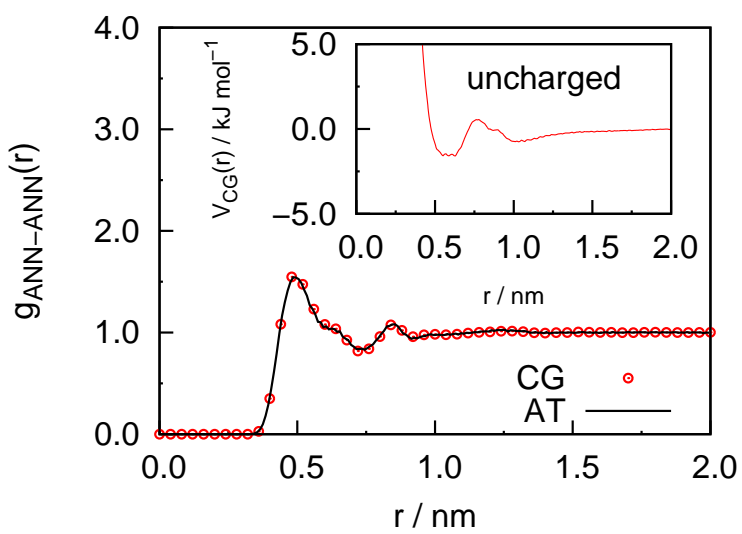

FIG. 8. The anion-anion radial distribution profile obtained from atomistic simulation (AT) and coarse-grained (CG) simulation as a function of distance $r$. The CG simulation employs the IBI potential for the uncharged CG model (inset).

tion of the system at the second order, where the order refers to the reduction of the $3 \mathrm{~N}$ probability distribution function to a factorized function of independent two-body terms (see $\operatorname{also}^{29}$ ). If the radial distribution functions of the atomistic region of GC-AdResS reproduce the behavior of the same quantities calculated in the sub region of a full atomistic simulation, then the two sub-regions are statistically equivalent at least up to the second order. That is, within such an accuracy, the atomistic degrees of freedom outside the sub-region are not relevant for determining quantities and properties of such a sub-region. In fact the atom-atom radial distribution functions of the hybrid region cannot reproduce the radial distribution function of the corresponding full atomistic simulation of reference $\left(\mathrm{sec}^{29}\right)$. Thus if atom-atom radial distribution functions in the AT region of AdResS reproduce the results of the reference full atomistic simulation, it implies that the only relevant atomistic degrees of freedom are those of the AT region. In practice the agreement between GC-AdResS and the full atomistic simulation regarding the local radial distribution functions tell us that the ensemble average of quantities depending on spatial coordinates can be localized in the sub-region with an accuracy at least up to the second order in the probability distribution. It has been shown that the second order is sufficient in MD to have a high accuracy for several relevant quantities and properties (see Ref. 29 ). 


\section{Consistency of GC-AdResS as a open boundary system}

Figure 9 shows the particle number density of ions pairs as a function of the distance from the center of the atomistic region. We have considered three different atomistic regions of radius $1.5 \mathrm{~nm}, 2.0 \mathrm{~nm}$ and $3.0 \mathrm{~nm}$ respectively. The results reported in Fig.9 show that the behavior of the ion pairs density for each atomistic region considered in GC-AdResS is satisfactory, independently from the coarse-grained model. Such an accuracy implies that the overall thermodynamic state point $(\rho, \mathrm{T})$ of the GC-AdResS simulations is the same as that of the reference full atomistic simulation within acceptable bounds.

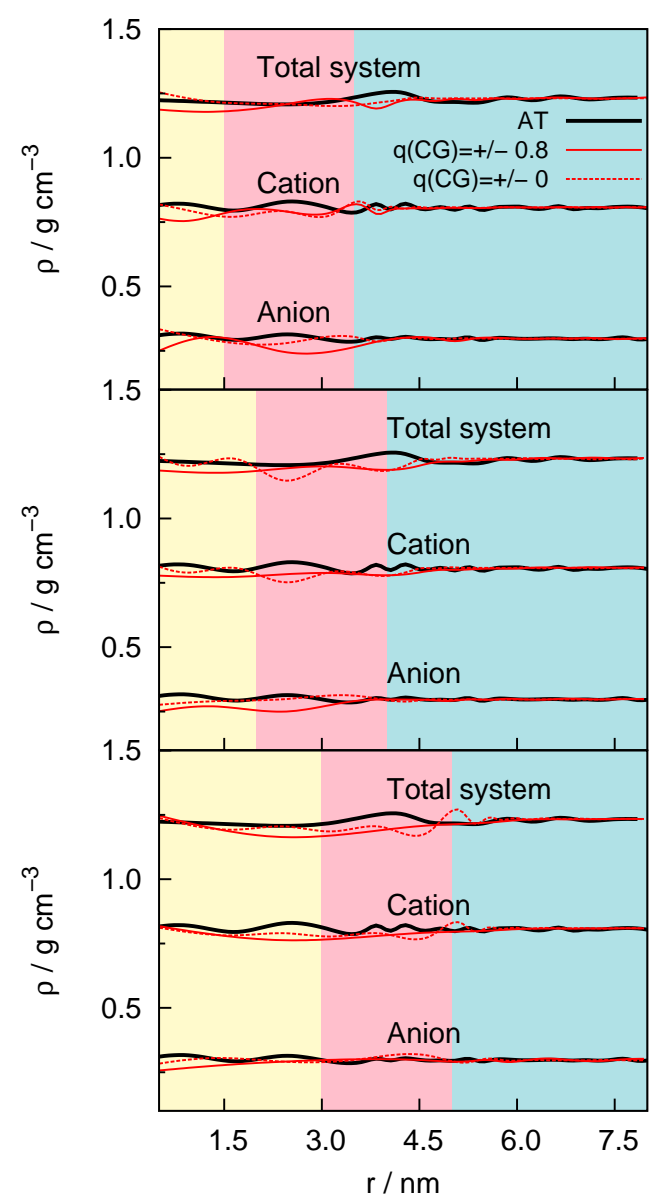

FIG. 9. The particle number density of ions pairs as a function of the distance from the center of the atomistic region. The yellow, pink and blue colors corresponds to atomistic, hybrid and coarse grained regions in the AdResS simulation. The experimental density of [Emim] [cl] is $1.2 \mathrm{~g} / \mathrm{cm}^{3} \underline{\underline{45}}$.

Figure 10] shows the probability distribution of the ion pairs number density in the atom- 
istic region of the three AdResS simulations compared with the results obtained for the corresponding sub-regions of the full atomistic simulation. The systematic shift in the location of the maxima, w.r.t. the full atomistic case, is due to the accuracy chosen for the thermodynamic force. We have chosen a threshold of $5 \%$ and in fact the largest shift in Figure 10 does not go beyond the threshold chosen. Our choice of $5 \%$ is based on the experience of previous studies, where we have seen that such an accuracy is sufficient for obtained satisfactory results for structural properties in the atomistic region (see for example the discussion about accuracy of density in Refs. $\underline{\underline{46}} \underline{\underline{47}})$. If a higher accuracy is needed, it would be sufficient to run the thermodynamic force with stricter criteria of convergence. The results of Figure 10 show a proper behavior of the probability distribution, which implies that the statistical exchange of molecules with the reservoir consisting of the hybrid and coarse-grained region occurs according to the expectations of a well behaving system with open boundaries. As for the density, the satisfactory character of the agreement with reference data does not depend strongly on the coarse-grained model. In fact the results obtained with the two model show differences which are within the accuracy of the calculations.

Finally, Figure 11 shows the probability distribution of the averaged order parameter $|\cos \theta|$, where $\theta$ is defined as the angle between the symmetry vector of the cation and the radial unit vector $\hat{r}$ with origin at the center of the spherical atomistic region. Also in this case the agreement is satisfactory. The charged coarse-grained model for $1.5 \mathrm{~nm}$ and $2.0 \mathrm{~nm}$ is slightly more accurate that the uncharged model. However, the difference between the two cases is, in general, not size-able. Such results show that the artificial change of resolution along the radial direction does not imply an artificial alignment of the cations due to the hybrid interface. The calculation of the quantities reported in this section assures as that the three subsystems we are considering in GC-AdResS properly behave at the relevant statistical and structural level and thus legitimate to go a step forward and perform the analysis of locality on more detailed and specific quantities, that is the radial distributions functions.

\section{Probing spatial locality}

As anticipated above, in order to probe the degree of spatial locality we calculate various radial distribution functions since they represent the $3 N$-probability distribution function 

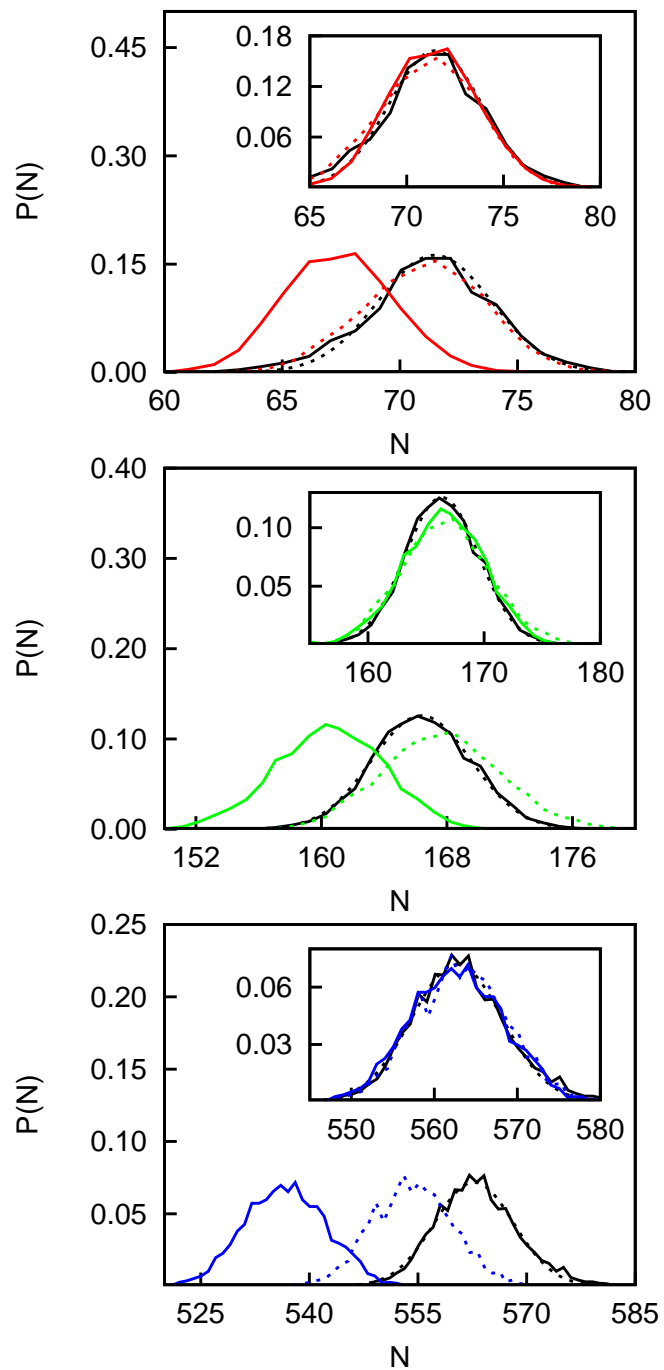

FIG. 10. The probability distribution of the ion pairs number density in the atomistic region of the three AdResS simulations (top 1.5nm, middle $2 \mathrm{~nm}$ and bottom 3nm) compared with the corresponding quantity in the equivalent sub-region of the full atomistic simulation. The charged and neutral CG models are represented in lines and dashed lines respectively. The distribution profile for the reference full atomistic system is shown in black solid line. The Gaussian fit for the reference system is shown in black dashed line. The location of the maximum of the distribution obtained in GC-AdResS simulations is shifted compared to the full atomistic reference. In fact we have set the accuracy of our calculations,through the thermodynamic force, at $5 \%$ and the maximum shift found is below $5 \%$. In such a case what is more relevant is the Gaussian shape of the function and from the insets, where the functions are over-imposed to the Gaussian fit, one can see that the agreement is satisfactory. 


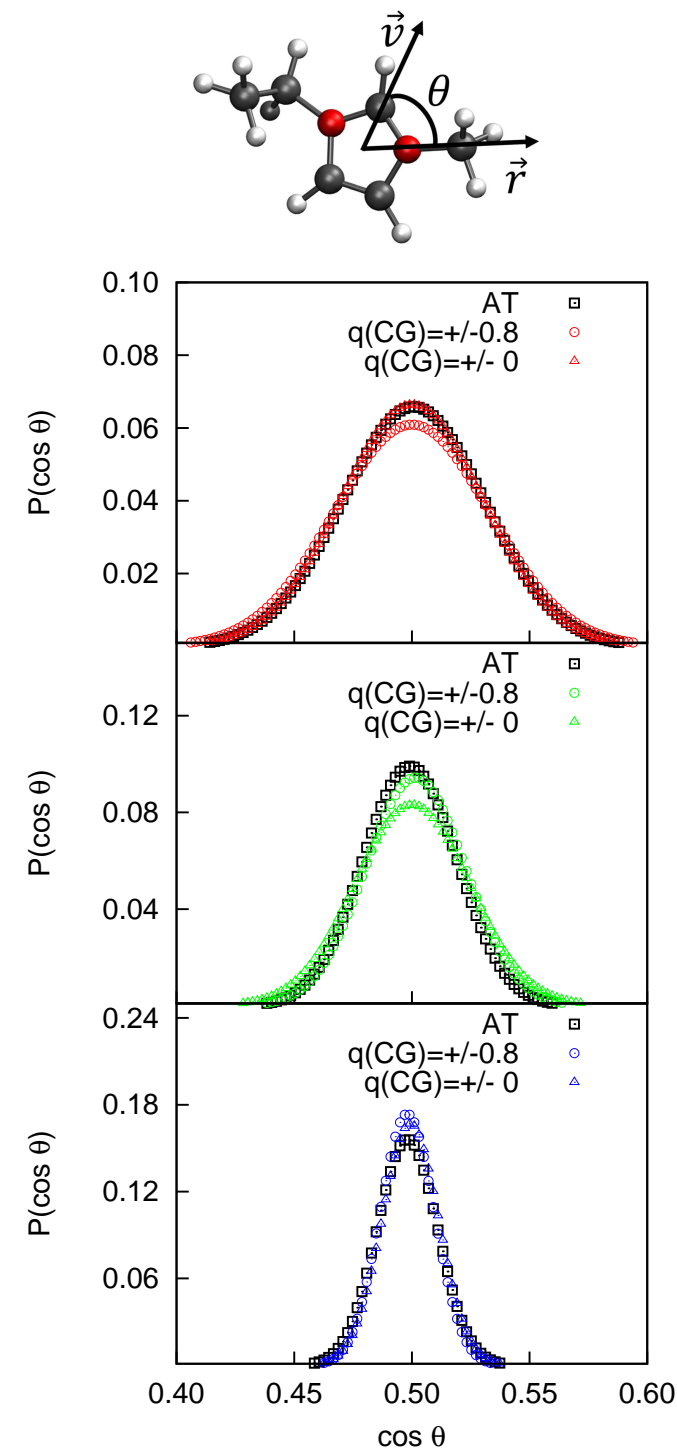

FIG. 11. The probability distribution of the averaged order parameter $|\cos \theta|$ (top panel) in the atomistic region of the three AdResS simulations (top $1.5 \mathrm{~nm}$, middle $2 \mathrm{~nm}$ and bottom $3 \mathrm{~nm}$ ) compared with the corresponding quantity in the equivalent sub-region of the full atomistic simulation

of the system at the second order. Moreover, the functions considered here are not only those defined by centers of mass of the cations but also those defined between different specific atoms. This means that they reproduce at the second order very detailed aspects of the $3 N$-probability distribution. Thus, if the atomistic region of GC-AdResS accurately reproduces such distributions then we infer that the atomistic degrees of freedom outside the atomistic region do not play a relevant role in the atomistic structure of such a region. 
As a consequence the calculation of space-dependent physical quantities will need only the knowledge of the local atomistic environment, i.e., the atomistic region.

Figures 12, 13, 14, 15, 16, 17, show results of various radial distribution functions. The different coarse-grained models, as already seen for the other quantities in the previous section, do not play a key role. The results show that for an atomistic region of $3.0 \mathrm{~nm}$ the agreement with the reference full atomistic simulation is rather satisfactory and that we can safely take $3.0 \mathrm{~nm}$ as the upper bound of locality. However, even for $1.5 \mathrm{~nm}$ the deviation from the data of reference is not dramatic, so that, although with somewhat reduced accuracy, even an atomistic region of $1.5 \mathrm{~nm}$ should be sufficient for the purpose. This result seems interesting because $1.5 \mathrm{~nm}$ corresponds to the size of few EMIM cations (Figure10 shows that there are only 70 ion pairs) and thus the level of locality is considerable.

Note also that similar results are obtained with a coarse-grained model involving uncharged particles. As a consequence, the idea of explicit charge-charge (atom-atom) long range electrostatics playing a specific key role in the atomistic structure of the liquid seems refuted. In any case, it must be also added that even for a CG model with neutral sites, the long-range feature of the electrostatic interaction is effectively incorporated in the CG potentials, therefore, a good reproduction of structural properties by a CG model with neutral sites cannot defeat the general importance of the long-range feature of the electrostatic interaction in IL systems, but, as specified above, it can only point out that the corresponding (explicit) charge atomistic degrees of freedom are not relevant. In the next section we discuss this point further.

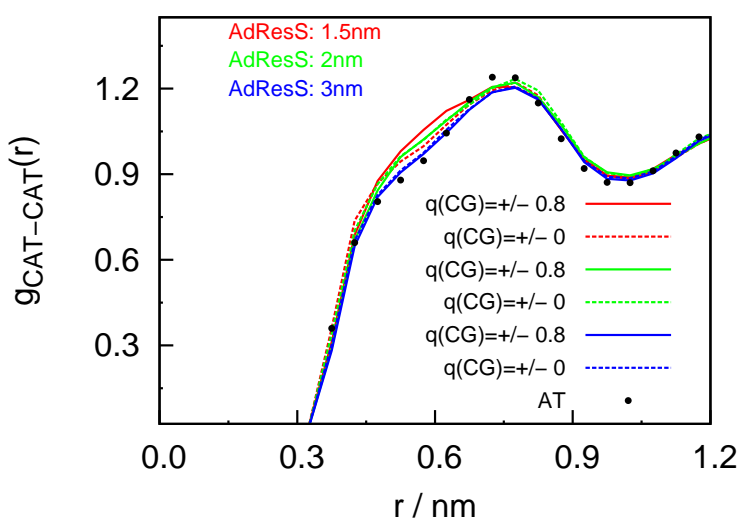

FIG. 12. The variation of the cation-cation radial distribution profile calculated in the atomistic region of AdResS for both coarse-grained models and compared with the corresponding quantity calculated in the equivalent region of a full atomistic simulation. 


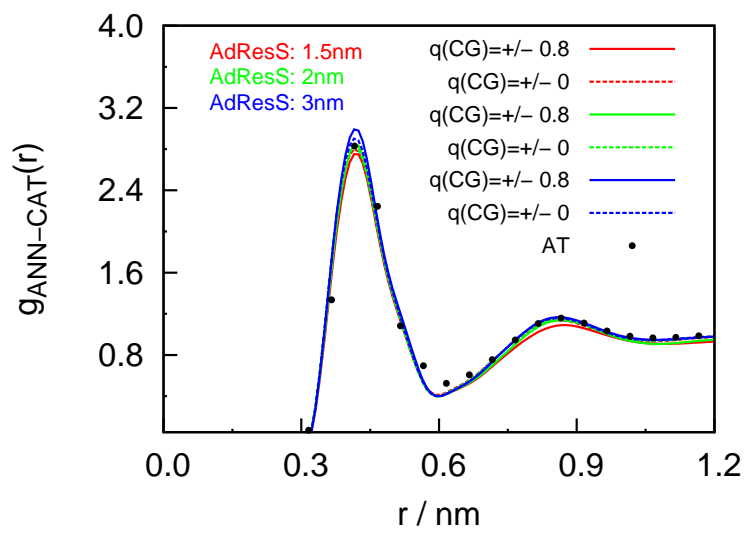

FIG. 13. The anion-cation radial distribution function calculated in the atomistic region of AdResS for both coarse-grained models and compared with the corresponding quantity calculated in the equivalent region of a full atomistic simulation.

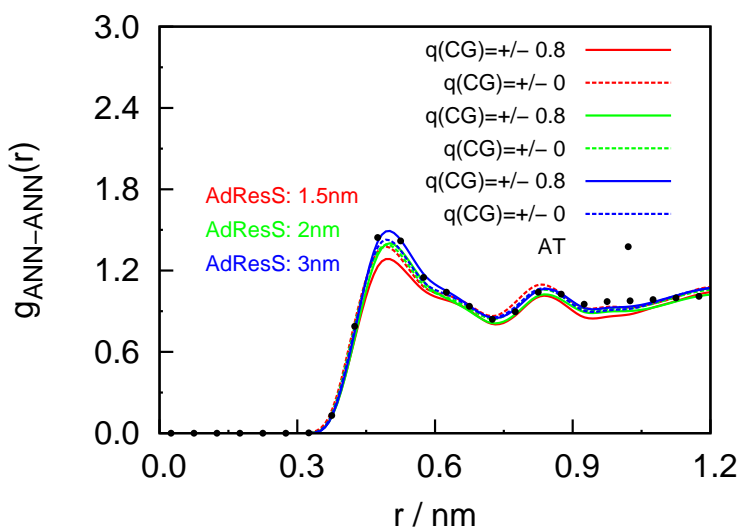

FIG. 14. The anion-anion radial distribution function calculated in the atomistic region of AdResS for both coarse-grained models and compared with the corresponding quantity calculated in the equivalent region of a full atomistic simulation.

\section{A. Modeling Perspectives}

The results shown in previous section suggest an overall scenario as that pictorially illustrated in Figure 18. One can reduce the model of an IL to the essential aspects by defining open atomistic islands centered around an anion and a cation. These islands are embedded in a macroscopic environment characterized only by macroscopic quantities, e.g. temperature, pressure, density, viscosity etc etc.. The representation of Figure 18 resembles closely the idea of ILs characterized by the rattling of ions in ion cages proposed in 


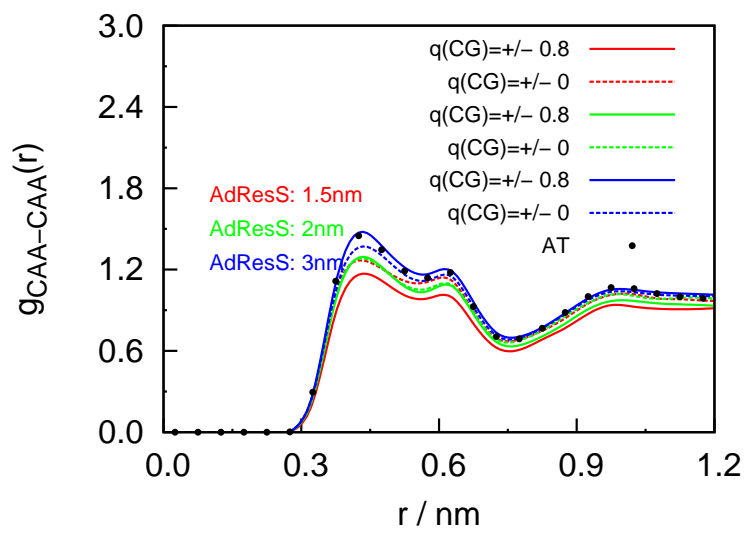

FIG. 15. The carbon-carbon radial distribution profile calculated in the atomistic region of AdResS for both coarse-grained models and compared with the corresponding quantity calculated in the equivalent region of a full atomistic simulation.

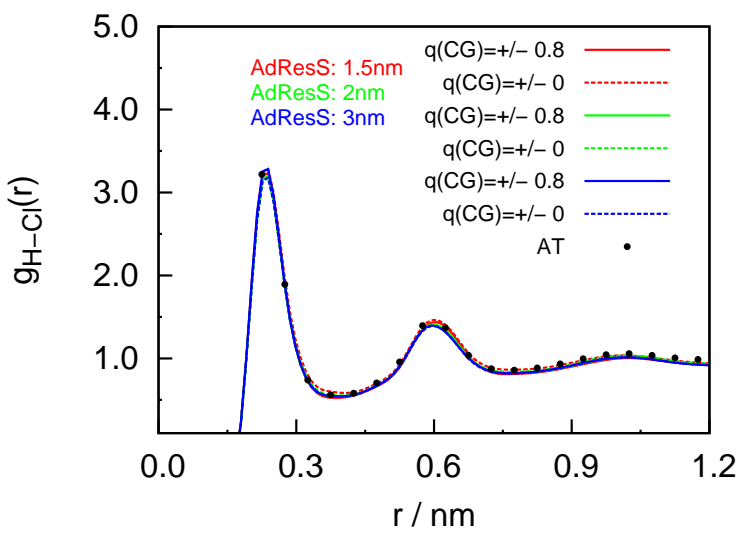

FIG. 16. The hydrogen-chloride radial distribution function calculated in the atomistic region of AdResS for both coarse-grained models and compared with the corresponding quantity calculated in the equivalent region of a full atomistic simulation.

the literature $\underline{\underline{48}} \underline{-52}$. Our method allows us to go even further and define the minimal size of the cages. In general our findings provide justification to a strategy of study based on reducing the atomistic analysis to small regions (for atomistic properties) and only consider the link of such regions with the reservoir for properties at a larger scale. This kind of analysis would be extremely useful for designing ILs at the molecular level specified by clear structure-function relations. Our findings also strengthen the conclusions of previous theoretical work regarding the general local character in space of ionic liquids $\frac{19,53}{}$. It suggests that by defining these spherical atomistically independent regions one can build physically well founded coarse-grained models of units larger than the molecular size and containing 


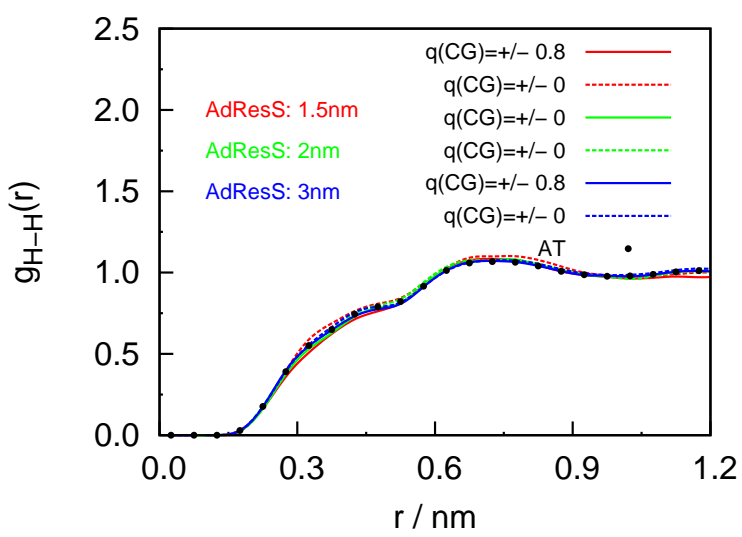

FIG. 17. The hydrogen-hydrogen radial distribution function calculated in the atomistic region of AdResS for both coarse-grained models and compared with the corresponding quantity calculated in the equivalent region of a full atomistic simulation.

an entire group of anions and cations. An example would be the solvation environment for an anion and cation sketched in Figure18, Such an idea has already been successfully used for other systems such as liquid water $\underline{\underline{54}}$.

\section{CONCLUSION}

We have tested the spatial locality of 1-ethyl 3-methyl imidazolium chloride liquid by employing the Grand Canonical Adaptive Resolution Molecular Dynamics technique. By calculating various radial distribution functions between anions and cations as a function of the size of the atomistic region and comparing them with the equivalent quantities in a full atomistic simulation we could conclude that even for a spherical region of radius $1.5 \mathrm{~nm}$ the atomistic degrees of freedom of the bulk do not play a relevant role. Our study can be seen in different perspectives (a) as a further argument that ionic liquids possess an inherent locality in the spatial structure, a hypothesis put forward in previous work ${ }^{19,53}$, and (b) as a modeling tool for designing ILs at the molecular level in the framework of structure-function relations. However, it must be underlined that our conclusions for other ionic liquids at this stage can only be qualitative and used a starting point for further studies. In fact the current systems is characterized by a relatively short alkyl side chain, thus the level of locality may change in a significant way for systems with longer alkyl chains. In this perspective, the next step will consist of repeating this analysis for a systematic comparison between different ILs aiming at understanding how a chemical modifications (e.g. the length of the of alkyl side 


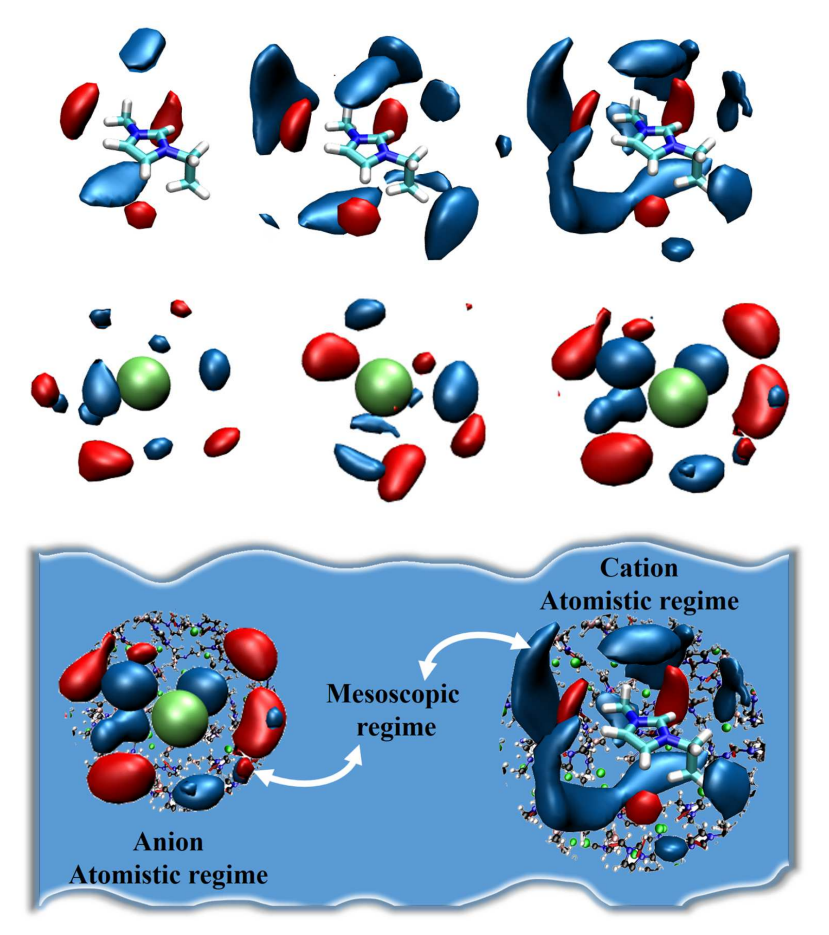

FIG. 18. Isosurfaces of the spatial probability density of anions (red) and cations (blue) as a function of the size of the atomistic regions (from $1.5 \mathrm{~nm}$ (left) to $3 \mathrm{~nm}$ (right)) in GC-AdResS simulations (with charge). The top and middle panels show the solvation environment of ions around the centered cations and anions, respectively. The bottom panel reports a pictorial representation of the essential solvation environment in space.

chain in the cations) influence the larger scale behavior of the liquid and, in particular, its degree of locality. This study will be considered in future publications.

Further interesting future perspectives may be offered by a recent proposal of GC-AdResS scheme for molecules with electrons, treated at the quantum level $\underline{55}$. Such an approach may pave the way for direct tests of locality at the very accurate chemical level and thus the open the possibility of a precise chemical manipulation of the molecules and a direct testing of its consequences within a larger scale environment.

\section{ACKNOWLEDGMENT}

This research has been funded by Deutsche Forschungsgemeinschaft (DFG) through grants CRC 1114 "Scaling Cascades in Complex Systems", project C01. This work has also received funding from the European Union's Horizon 2020 research and innovation program under the grant agreement No. 676531 (project E-CAM). We would like to thank 
Barbara Kirchner for a critical reading of the manuscript and the members of her group for providing technical assistance regarding the choice and implementation of the force fields used. 


\section{REFERENCES}

${ }^{1}$ R. Shi and Y. Wang, "Dual Ionic and Organic Nature of Ionic Liquids," Scientific Reports 6, 19644 (2016).

${ }^{2}$ N. V. Plechkova and K. R. Seddon, "Applications of Ionic Liquids in the Chemical Industry," Chem. Soc. Rev. 37, 123-150 (2008).

${ }^{3}$ P. Hapiot and C. Lagrost, "Electrochemical Reactivity in Room-Temperature Ionic Liquids," Chem. Rev. 108, 2238-2264 (2008).

${ }^{4}$ R. Giernoth, "Task-Specific Ionic Liquids," Angew. Chem., Int. Ed. 49, 2834-2839 (2010).

${ }^{5}$ A. Sawant, D. Raut, N. Darvatkar, and M. Salunkhe, "Recent Developments of TaskSpecific Ionic Liquids in Organic Synthesis," Green Chem. Lett. Rev. 4, 41-54 (2011).

${ }^{6}$ Y. Sahbaz, H. D. Williams, T. Nguyen, J. Saunders, L. Ford, S. A. Charman, P. J. Scammells, and C. J. H. Porter, "Transformation of Poorly Water-Soluble Drugs into Lipophilic Ionic Liquids Enhances Oral Drug Exposure from Lipid Based Formulations," Mol. Pharmaceutics 12, 1980-1991 (2015).

${ }^{7}$ M. L. Hart, D. P. Do, R. A. Ansari, and S. A. Rizvi, "Brief Overview of Various Approaches to Enhance Drug Solubility," J. Dev. Drugs 2, 1000110-1000116 (2013).

${ }^{8}$ M. Lin, M. Gong, B. Lu, Y. Wu, D. Wang, M. Guan, M. Angell, C. Chen, J. Yang, B. Hwang, and et al., "An Ultrafast Rechargeable Aluminium-Ion Battery," Nature 520, 324-328 (2015).

${ }^{9}$ G. M. A. Girard, M. Hilder, H. Zhu, D. Nucciarone, K. Whitbread, S. Zavorine, M. Moser, M. Forsyth, D. R. MacFarlane, and P. C. Howlett, "Electrochemical and Physicochemical Properties of Small Phosphonium Cation Ionic Liquid Electrolytes with High Lithium Salt Content," Phys. Chem. Chem. Phys. 17, 8706-8713 (2015).

${ }^{10}$ D. M. Piper, T. Evans, K. Leung, T. Watkins, J. Olson, S. C. Kim, S. S. Han, V. Bhat, K. H. Oh, D. A. Buttry, and et al., "Stable Silicon-Ionic Liquid Interface for NextGeneration Lithium-Ion Batteries," Nat. Commun. 6, 6230 (2015).

${ }^{11}$ C. Zhang, A. Yamazaki, J. Murai, J. Park, T. Mandai, K. Ueno, K. Dokko, and M. Watanabe, "Chelate Effects in Glyme/Lithium Bis-(Trifluoromethanesulfonyl)Amide Solvate Ionic Liquids, Part 2: Importance of Solvate-Structure Stability for Electrolytes of Lithium Batteries," J. Phys. Chem. C 118, 17362-17373 (2014). 
${ }^{12}$ M. Taha, M. R. Almeida, F. A. Silva, P. Domingues, S. P. M. Ventura, J. A. P. Coutinho, and M. G. Freire, "Novel Biocompatible and Self-Buffering Ionic Liquids for Biopharmaceutical Applications," Chem. -Eur. J. 21, 4781-4788 (2015).

${ }^{13}$ J. Gorke, F. Srienc, and R. Kazlauskas, "Toward Advanced Ionic Liquids. Polar, EnzymeFriendly Solvents for Biocatalysis," Biotechnol. Bioprocess Eng. 15, 40-53 (2010).

${ }^{14}$ A. M. Socha, R. Parthasarathi, J. Shi, S. Pattathil, D. Whyte, M. Bergeron, A. George, K. Tran, V. Stavila, S. Venkatachalam, and et al., "Efficient Biomass Pretreatment Using Ionic Liquids Derived from Lignin and Hemicellulose," Proc. Natl. Acad. Sci. U. S. A. 111, E3587-E3595 (2014).

${ }^{15}$ H. Wang, G. Gurau, and R. D. Rogers, "Ionic Liquid Processing of Cellulose," Chem. Soc. Rev. 41, 1519-1537 (2012).

${ }^{16}$ T. Mendez-Morales, J. Carrete, O. Cabeza, L. J. Gallego, and L. M. Varela, "Molecular Dynamics Simulation of the Structure and Dynamics of Water-1-Alkyl-3methylimidazolium Ionic Liquid Mixtures," J. Phys. Chem. B 115, 6995-7008 (2011).

${ }^{17}$ H. Luo, S. Dai, P. V. Bonnesen, and A. C. Buchanan III, "Separation of Fission Products Based on Ionic Liquids: Task-Specific Ionic Liquids Containing an Aza-Crown Ether Fragment," J. Alloy. Compd. 418, 195-199 (2006).

${ }^{18} \mathrm{~S}$. M. Urahata and M. C. C. Ribeiro, "Structure of Ionic Liquids of 1- Alkyl-3Methylimidazolium Cations: A Systematic Computer Simulation Study," J. Chem. Phys. 120, 1855 (2004).

${ }^{19}$ Y. Wang and G. A. Voth, "Unique Spatial Heterogeneity in Ionic Liquids," J. Am. Chem. Soc. 127, 12192-12193 (2005).

${ }^{20}$ J. N. A. Canongia Lopes and A. A. H. Padua, "Nanostructural Organization in Ionic Liquids," J. Phys. Chem. B 110, 3330-3335 (2006).

${ }^{21}$ Y. Wang and G. A. Voth, "Tail Aggregation and Domain Diffusion in Ionic Liquids," J. Phys. Chem. B 110, 18601-18608 (2006).

${ }^{22}$ A. Triolo, O. Russina, H.-J. Bleif, and E. Di Cola, "Nanoscale Segregation in Room Temperature Ionic Liquids," J. Phys. Chem. B 111, 4641-4644 (2007).

${ }^{23}$ J. Schmidt, C. Krekeler, F. Dommert, Y. Zhao, R. Berger, L. Delle Site, and C. Holm, "Ionic Charge Reduction and Atomic Partial Charges from First-Principles Calculations of 1,3-Dimethylimidazolium Chloride," J. Phys. Chem. B 114, 6150-6155 (2010). 
${ }^{24}$ C. Krekeler, F. Dommert, J. Schmidt, Y. Y. Zhao, C. Holm, R. Berger, and L. Delle Site, "Electrostatic Properties of Liquid 1,3-Dimethylimidazolium Chloride: Role of Local Polarization and Effect of the Bulk," Phys. Chem. Chem. Phys. 12, 1817-1821 (2010).

${ }^{25}$ K. Wendler, S. Zahn, F. Dommert, R. Berger, C. Holm, B. Kirchner, and L. Delle Site, "Locality and Fluctuations: Trends in Imidazolium-Based Ionic Liquids and Beyond," J. Chem. Theory Comput. 7, 3040-3044 (2011).

${ }^{26}$ M. Praprotnik, L. Delle Site, and K. Kremer, "Adaptive Resolution Molecular-Dynamics Simulation: Changing the Degrees of Freedom on the Fly," J. Chem. Phys. 123, 224106 (2005).

${ }^{27}$ M. Praprotnik, L. Delle Site, and K. Kremer, "Multiscale Simulation of Soft Matter: From Scale Bridging to Adaptive Resolution," Annu. Rev. Phys. Chem. 59, 545-571 (2008).

${ }^{28}$ H. Wang, C. Schütte, and L. Delle Site, "Adaptive Resolution Simulation (AdResS): A Smooth Thermodynamic and Structural Transition from Atomistic to Coarse Grained Resolution and Vice Versa in a Grand Canonical Fashion," J. Chem. Theory Comput. 8, 2878-2887 (2012).

${ }^{29}$ H. Wang, C. Hartmann, C. Schütte, and L. Delle Site, "Grand-Canonical-Like MolecularDynamics Simulations by Using an Adaptive-Resolution Technique," Phys. Rev. X 3, 011018 (2013).

${ }^{30}$ A. Agarwal, J. Zhu, C. Hartmann, H. Wang, and L. Delle Site, "Molecular Dynamics in a Grand Ensemble: Bergmann-Lebowitz Model and Adaptive Resolution Simulation," New J. Phys. 17, 083042 (2015).

${ }^{31}$ L. Delle Site and M. Praprotnik, "Molecular Systems with Open Boundaries: Theory and Simulation," Phys. Rep. 693, 1-56 (2017).

${ }^{32}$ B. Lambeth, C. Junghans, K. Kremer, C. Clementi, and L. Delle Site, "On the Locality of Hydrogen Bond Networks at Hydrophobic Interfaces," J. Chem. Phys. 133, 221101 (2010).

${ }^{33}$ C. Agarwal, A.and Clementi and L. Delle Site, "Path Integral-GC-AdResS Simulation of a Large Hydrophobic Solute in Water: A Tool to Investigate the Interplay Between Local Microscopic Structures and Quantum Delocalization of Atoms in Space ," Phys. Chem. Chem. Phys. 19, 13030-13037 (2017).

${ }^{34}$ C. Krekeler and L. Delle Site, "Towards Open Boundary Molecular Dynamics Simulation of Ionic Liquids," Phys. Chem. Chem. Phys. 19, 4701-4709 (2017). 
${ }^{35}$ M. Praprotnik, S. Matysiak, L. Delle Site, K. Kremer, and C. Clementi, "Adaptive Resolution Simulation of Liquid Water," J. Phys.: Condens. Matter 19, 292201 (2007).

${ }^{36}$ S. Poblete, M. Praprotnik, K. Kremer, and L. Delle Site, "Coupling Different Levels of Resolution in Molecular Simulations," J. Chem. Phys. 132, 114101 (2010).

${ }^{37}$ S. Fritsch, S. Poblete, C. Junghans, G. Ciccotti, L. Delle Site, and K. Kremer, "Adaptive Resolution Molecular Dynamics Simulation Through Coupling to an Internal Particle Reservoir," Phys. Rev. Lett. 108, 170602 (2012).

${ }^{38}$ L. Delle Site, "Formulation of Liouville's Theorem for Grand Ensemble Molecular Simulations," Phys. Rev. E 93, 022130 (2016).

${ }^{39}$ F. Dommert, K. Wendler, R. Bergerm, L. Delle Site, and C. Holm, "Force Fields for Studying the Structure and Dynamics of Ionic Liquids: A Critical Review of Recent Developments," ChemPhysChem 13, 1625 (2012).

${ }^{40}$ B. Hess, C. Kutzner, D. van der Spoel, and E. Lindahl, "GROMACS 4: Algorithms for Highly Efficient, Load-Balanced, and Scalable Molecular Simulation," J. Chem. Theory Comput. 4, 435 (2008).

${ }^{41}$ H. J. C. Berendsen, J. P. M. Postma, W. F. van Gunsteren, A. DiNola, and J. R. Haak, "Molecular Dynamics with Coupling to an External Bath," J. Chem. Phys. 81, 3684-3690 (1984).

${ }^{42}$ M. Parrinello and A. Rahman, "Polymorphic Transitions in Single Crystals: A New Molecular Dynamics Method," J. Appl. Phys. 52, 7182 (1981).

${ }^{43}$ D. Reith, M. Putz, and F. Muller-Plathe, "Deriving Effective Mesoscale Potentials from Atomistic Simulations," J. Comput. Chem. 24, 1624-1636. (2003).

${ }^{44}$ S. Fritsch, C. Junghans, and K. Kremer, "Structure Formation of Toluene Around C60: Implementation of the Adaptive Resolution Scheme (AdResS) into GROMACS," J. Chem. Theory Comput. 8, 398-403 (2012).

${ }^{45}$ A. V. Klinov, M. V. Fedorov, A. V. Malygin, and L. R. Minibaeva, "Properties of an Aqueous Solution of Ionic Liquid [Emim][Cl] at Standard Atmospheric Pressure," Russian J. Phys. Chem. A 88, 1497-1503 (2014).

${ }^{46}$ A. Agarwal and L. Delle Site, "Path Integral Molecular Dynamics within the Grand Canonical-like Adaptive Resolution Technique: Simulation of Liquid Water," J. Chem. Phys. 143, 094102 (2015). 
${ }^{47}$ J. Peters, R. Klein, and L. Delle Site, "Simulation of Macromolecular Liquids with the Adaptive Resolution Molecular Dynamics Technique," Phys. Rev. E 94, 023309 (2016).

${ }^{48} \mathrm{~S}$. Zahn, J. Thar, and B. Kirchner, "Structure and Dynamics of the Protic Ionic Liquid Monomethylammonium Nitrate ([CH3NH3][NO3]) from Ab-Initio Molecular Dynamics Simulations ," J. Chem. Phys. 132, 124506 (2010).

${ }^{49}$ T. I. Morrow and E. J. Maginn, "Molecular Dynamics Study of the Ionic Liquid 1-n-Butyl3-methylimidazolium Hexafluorophosphate," J. Phys. Chem. B 106, 1287 (2002).

${ }^{50}$ M. G. D. Popolo and G. A. Voth, "On the Structure and Dynamics of Ionic Liquids," J. Phys. Chem. B 108, 1744 (2008).

${ }^{51}$ D. A. Turton, J. Hunger, A. Stoppa, G. Hefter, M. W. A. Thoman, R. Buchner, and K. Wynne, "Dynamics of Imidazolium Ionic Liquids from a Combined Dielectric Relaxation and Optical Kerr Effect Study: Evidence for Mesoscopic Aggregation," J. Am. Chem. Soc. 131, 11140 (2009).

${ }^{52}$ T. Mendez-Morales, J. Carrete, O. Cabeza, J. L. Gallego, and L. M. Varela, "Molecular Dynamics Simulation of the Structure and Dynamics of Water1-Alkyl-3methylimidazolium Ionic Liquid Mixtures," J. Phys. Chem. B 115, 6995 (2011).

${ }^{53}$ K. Wendler, S. Zahn, F. Dommert, R. Berger, C. Holm, B. Kirchner, and L. Delle Site, "Locality and Fluctuations: Trends in Imidazolium-Based Ionic Liquids and Beyond," J. Chem. Theory Comput. 7, 3040-3044 (2011).

${ }^{54}$ J. Zavadlav and M. Praprotnik, "Adaptive Resolution Simulations Coupling Atomistic Water to Dissipative Particle Dynamics," J. Chem. Phys. 147, 114110 (2017).

${ }^{55}$ L. Delle Site, "Grand Canonical Adaptive Resolution Simulation for Molecules with Electrons: A Theoretical Framework based on Physical Consistency," Comp. Phys. Comm. (2017), https://doi.org/10.1016/j.cpc.2017.09.020. 